\title{
The Application of Recursive Least Squares in Synchronous Generator Parameter Identification
}

\author{
Huiying Huang ${ }^{1} *$ and Juanmei Zhang $^{2}$ \\ ${ }^{1}$ Department of Electrical Engineering and Automation, Luoyang Institute of \\ Science and Technology, Luoyang471023, China. \\ ${ }^{2}$ Department of Electrical Engineering and Automation, Luoyang Institute of \\ Science and Technology, Luoyang471023, China. \\ hjfhhy1@163.com, 2 smxjixin@163.com \\ Abstract
}

\begin{abstract}
The paper took Park model of synchronous generator as is research object, derivated the space model of synchronous generator on magnetic chain which/can transform with transfer function, make for the parameter calculate and identification. On the side, based on system identification theory, the paper proposes a method of synchronous generator parameter identification based on recursive teast squares, wo can prove its astringency in theory. Finally, program on the model parameter estimation, and simulate a synchronous generator of known parameters. The parametens' errors are identified to conform to design requirements, which show the high identifioation accuracy and stability of the method.
\end{abstract}

Keywords: Synchronous generaton Recursive least squares (RLS), Parameter identification, MATLAB

\section{Introduction}

The economic efficiencly of the power system has been greatly improved, under the rapid development of capacity and network scale of modern power systems, but the factors of restricting safety and reliable operation are growing[1]. The accuracy of generating dynanic parameters is directly related to the stable operation of the system, in many factors. Second, the transient voltage waveform synchronous generator, the calculates of the motor impulse voltage and the design of excitation system of the motor requireal-accurate transient parameters[2]. The accuracy of synchronous generator parameters is of great importance for power system analysis.

The accurate and suitable mathematical model is must, in addition, the selection algorithm is very importantwhen we are identifying for the parameter of synchronous generator [3]. In fact, Parameter identification of synchronous generator is a process of a parameter optimization, which aims to make the system output in the identification value approaching to the actual output [4]. Aiming at the characteristics of synchronous generator model, the paper analyzed the feasibility of recursive least squares parameter identification method of synchronous generator circuit, focused on the state space equations and recursive least squares identification step synchronous generator [5]. Because the model is the state equation model. We need to change it into a transfer function model for identification, it is necessary to state equation model and transfer function model for conversion, finally the article successfully solved this problem and achieved the identification process[6]. 


\section{The Equation State Model}

The numerous studies have shown that the parameters accuracy is not high utility, stability is not good of synchronous generator parameters identification based on utility model [7].Therefore, the paper proposes Park model with parameter identification of synchronous generator.

\subsection{The Park Model of Synchronous Generator}

Park transform converted the synchronous generator geostationary space coordinates a, $\mathrm{b}, \mathrm{c}$ into dynamic $\mathrm{d}, \mathrm{q}, 0$ coordinate system, the resulting equations are a set of nonlinear ordinary differential equations set, called Parker equation. Because of irrelevance between d, q, 0 coordinate triaxial and constant inductance parameters reasons, Pike equations are widely used in the parametric analysis of synchronous generator [8-10].

Synchronous generator voltage equation and flux equations (in the form of unit value) are as follows:

1. Voltage equation matrix:

2. Flux equation matrix:

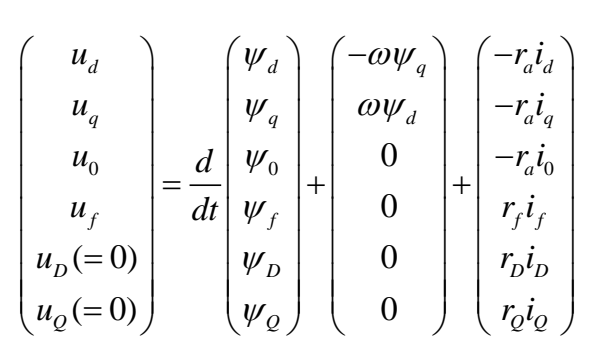

$$
\left(\begin{array}{c}
\psi_{d} \\
\psi_{q} \\
\psi_{0} \\
\psi_{f} \\
\psi_{D} \\
\psi_{\psi_{Q}}
\end{array}\right)=\left(\begin{array}{cccccc}
X_{d} & 0 & 0 & X_{a d} & X_{a d} & 0 \\
0 & X_{q} & 0 & 0 & 0 & X_{a q} \\
0 & 0 & X_{0} & 0 & 0 & 0 \\
X_{a d} & 0 & 0 & X_{f} & X_{a d} & 0 \\
X_{a d} & 0 & 0 & X_{a d} & X_{D} & 0 \\
0 & X_{a q} & 0 & 0 & 0 & X_{Q}
\end{array}\right)\left(\begin{array}{c}
-i_{d} \\
-i_{q} \\
-i_{0} \\
i_{f} \\
i_{D} \\
i_{Q}
\end{array}\right)
$$

Meaning of each cemponent as follows:

$i_{d}$ :D-axis component of the load current; $i_{q}: \mathrm{q}$-axis component of the load current; $i_{f}$ : excitation current: $y_{d}$ :terminal voltage d-axis component; $u_{q}: \mathrm{q}$-axis component of the terminal voltage; $u_{f}$ :the field winding voltage; $r_{f}$ :the field winding resistance; $i_{D}$ :direct axis damper winding currents ; $i_{Q}$ :Cross-axis damper winding current; $X_{\mathrm{d}}$ :direct axis reactance; $X_{a d}$ : direchaxis reaction reactance; $X_{q}$ :quadrature axis reactance; $X_{a q}$ :cross-axis reaction reactance, $X_{D}$ : direct axis damper winding reactance; $X_{Q}$ :cross-axis damper winding reactance; $\Psi_{d}$ :direct axis flux; $\Psi_{q}$ :cross-axis flux; $\Psi_{f}$ :the field winding flux; $\Psi_{D}$ :direct axis damper winding flux; $\Psi_{Q}$ :cross-axis damper winding flux.

\subsection{The State Space Model of Synchronous Generator}

The studied turbogenerator have high speed in the paper, centrifugal force limit, using the characteristics of hidden structure the rotor pole[11]. $\quad X_{d}=X_{q}$ So, $X_{a d}=X_{a q}$ and vertical and horizontal axes of the leakage reactance is equal, it is assumed $X_{l}$. to be 
Assumed

$$
\left\{\begin{array}{l}
X_{d}-X_{a d}=X_{l} \\
X_{q}-X_{a q}=X_{l} \\
X_{D}-X_{a d}=X_{D l} \\
X_{Q}-X_{a q}=X_{Q l} \\
X_{f}-X_{a d}=X_{f l}
\end{array}\right.
$$

And assumed

$$
\left\{\begin{array}{c}
\frac{1}{X_{m d}}=\frac{1}{X_{a d}}+\frac{1}{X_{l}}+\frac{1}{X_{f l}}+\frac{1}{X_{D l}} \\
\frac{1}{X_{m q}}=\frac{1}{X_{a q}}+\frac{1}{X_{l}}+\frac{1}{X_{Q l}}
\end{array}\right.
$$

The conclusion can be introduced by the flux equation

$$
\begin{aligned}
&\left\{\begin{array}{l}
\psi_{d}-\psi_{f}=-X_{l} i_{d}-X_{f l} i_{f} \\
\psi_{f}-\psi_{D}=X_{f} i_{f}-X_{D l} i_{D} \\
\psi_{D}-\psi_{d}=X_{l} i_{d}+X_{D l} i_{D}
\end{array}\right. \\
& \psi_{d}+\psi_{f}+\psi_{D}=-\left(3 X_{a d}+X_{l}\right) i_{d}+\left(3 X_{a d}+X_{f f}\right) l_{f} \pm\left(3 X_{a d}+X_{D l}\right) i_{B}
\end{aligned}
$$

$i_{d} 、 i_{f} 、 i_{D}$ can be introduced from (1) (2)formula, which is

Similarly, you can latunch. Which is $i_{q}, i_{Q}$

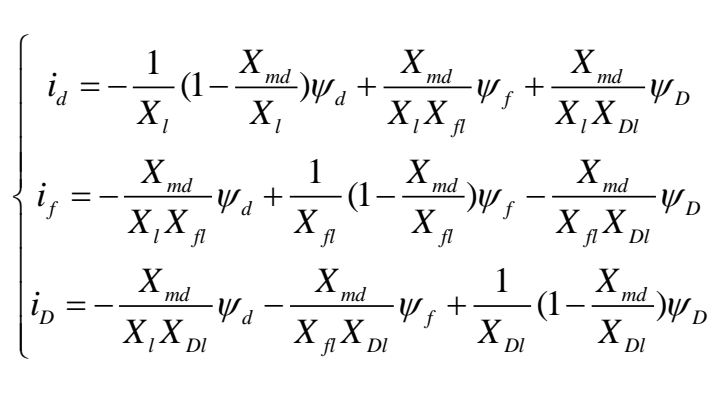

Substituting (3), (4) formula into synchronous generator voltage equations model, flux can be oblained from state variables .

$$
\left\{\begin{array}{c}
\dot{\psi}=A(\alpha) \psi(\alpha)+u \\
i=C(\alpha) \psi(\alpha)
\end{array}\right.
$$

System matrix is:

$$
A(\alpha)=\left[\begin{array}{ccccc}
-r_{a} A_{11} & r_{a} A_{12} & r_{a} A_{13} & \omega & 0 \\
-r_{f} A_{21} & -r_{f} A_{22} & -r_{f} A_{23} & 0 & 0 \\
r_{D} A_{31} & r_{D} A_{32} & r_{D} A_{32} & 0 & 0 \\
-\omega & 0 & 0 & r_{a} A_{44} & r_{a} A_{45} \\
0 & 0 & 0 & r_{Q} A_{54} & r_{Q} A_{55}
\end{array}\right]
$$


Output matrix is:

Each component as follows:

$$
C(\alpha)=\left[\begin{array}{ccccc}
A_{11} & A_{12} & A_{13} & 0 & 0 \\
A_{21} & A_{22} & A_{23} & 0 & 0 \\
0 & 0 & 0 & A_{44} & A_{45}
\end{array}\right]
$$

$$
\begin{aligned}
& A_{11}=-\frac{1}{X_{l}}\left(1-\frac{X_{m d}}{X_{l}}\right) A_{12}=\frac{X_{m d}}{X_{l} X_{f l}} A_{13}=\frac{X_{m d}}{X_{l} X_{D l}} \\
& A_{21}=-\frac{X_{m d}}{X_{l} X_{f l}} A_{22}=\frac{1}{X_{f l}}\left(1-\frac{X_{m d}}{X_{f l}}\right) A_{23}=-\frac{X_{m d}}{X_{f l} X_{D l}} \\
& A_{31}=\frac{X_{m d}}{X_{l} X_{D l}} A_{32}=\frac{X_{m d}}{X_{f l} X_{D l}} A_{33}=-\frac{1}{X_{D l}}\left(1-\frac{X_{m d}}{X_{D l}}\right)
\end{aligned}
$$

$$
\begin{aligned}
& \psi=\left[\begin{array}{lllll}
\psi_{d} & \psi_{f} & \psi_{D} & \psi_{q} & \psi_{Q}
\end{array}\right]^{T} \\
& A_{44}=-\frac{1}{X_{l}}\left(1-\frac{X_{m q}}{X_{l}}\right) A_{45}=\frac{X_{m q}}{X_{l} X_{Q l}} A_{54}=\frac{X_{m A}}{X_{l} X_{l l}} A_{55}-\frac{1}{X_{Q l}}
\end{aligned}
$$

State phasor

Control pharos

$$
u=\left[\begin{array}{lllll}
u_{d} & u_{f} & 0 & u_{q} & 0
\end{array}\right]^{T}
$$

Measuring phasor $\quad i=\left[\begin{array}{lll}i_{d} & i_{f} & i_{q}\end{array}\right]^{T}$

\section{Synchronous Generator Parameter Identification}

The least squares method is computationally simple, easy to understand of the principle, it has a faster convergence rate, characterrusticseasy to understand and program, and ithasnot any requirement for the statistica 1 properties of random variables ,it is very widely used in the system parameter estimates[12-13].

In the state estimationof power system, parameter identification and adaptive control, the least squares method has very broad application prospects.

\subsection{The Basic Theory of Least Squares Algorithm}

Seeking squared sum of the differences between models actually observed values and calculated values, when it is minimum; Substituting observed values into the model output equation, theooutput value of the actual process output value is obtained at this time close. Namely the least squares estimates is the parameter values make the objective function minimum.

\subsection{MMO Linear System Parameter Identification}

In the state-space model, when the number of unknown parameters is less than the length of the measurement data, the minimum error solution of the objective function is easy to find, the model parameters can be identified [14].For MIMO linear systems ( $r$ input $\mathrm{m}$ output), the transfer function matrix is:

$$
G(z)=\frac{1}{A\left(z^{-1}\right)}\left[\begin{array}{cccc}
B_{11}\left(z^{-1}\right) & B_{12}\left(z^{-1}\right) & \cdots & B_{1 r}\left(z^{-1}\right) \\
B_{21}\left(z^{-1}\right) & B_{22}\left(z^{-1}\right) & \cdots & B_{2 r}\left(z^{-1}\right) \\
\vdots & \vdots & \ddots & \vdots \\
B_{m 1}\left(z^{-1}\right) & B_{m 2}\left(z^{-1}\right) & \cdots & B_{m r}\left(z^{-1}\right)
\end{array}\right]
$$

In the above formula:

$$
\left\{\begin{array}{c}
A\left(z^{-1}\right)=1+a(1) z^{-1}+a(2) z^{-2}+\cdots+a\left(n_{a}\right) z^{-n_{a}} \\
B_{i j}\left(z^{-1}\right)=z^{-d_{i j}}\left[b_{i j}(0)+b_{i j}(1) z^{-1}+\cdots+b_{i j}\left(n_{b_{i j}}\right) z^{-n_{i j}}\right] \\
i=1,2, \cdots, m ; j=1,2, \cdots, r
\end{array}\right.
$$


The multivariable system can be described as

$$
A\left(z^{-1}\right) y(k)=B\left(z^{-1}\right) u(k)+A\left(z^{-1}\right) \xi(k)
$$

In the formula $\xi(k)=\left[\xi_{1}(k), \xi_{2}(k), \cdots \xi_{m}(k)\right]^{T}, \quad \xi_{i}(k)$ is White noise, and

$$
\left\{\begin{array}{c}
E\{\xi(k)\}=0 \\
\operatorname{Cov}\{\xi(k)\}=\sigma_{\xi}^{2} I_{m} \\
E\left\{\xi(k) \xi^{T}(j)\right\}=\sigma_{\xi}^{2} \delta_{k j} I_{m}
\end{array}\right.
$$

Multivariable system can be seen as composed by the $m$ independent univariate system components, the i-th sub-systems can be expressed as

$$
A\left(z^{-1}\right) y_{i}(k)=\sum_{j=1}^{r} B_{i j}\left(z^{-1}\right) u_{j}(k)+e_{i}(k) \quad i=1,2, \cdots, m
$$

Inthe eighth formula:

$$
e_{i}(k)=A\left(z^{-1}\right) \xi_{i}(k)
$$

Supposed: $\quad \theta=\left[a^{T}, \theta_{1}^{T}, \theta_{2}^{T}, \cdots, \theta_{m}^{T}\right]^{T}$

$$
\text { Among }\left\{\begin{array}{c}
a=\left[a(1), a(2), \cdots, a\left(n_{a}\right)\right]^{T} \\
\theta_{i}=\left[b_{i 1}^{T}, b_{i 2}^{T}, \cdots, b_{i r}^{T}\right]^{T} \\
b_{i j}=\left[b_{i j}(0), b_{i j}(1), \cdots, b_{i j}\left(n_{b i j}\right)\right]^{T}
\end{array}\right.
$$

$y(k)=\left[y_{1}(k), y_{2}(k), \cdots, y_{m}(k)\right]^{T} e(k)=\left[e_{1}(k), e_{2}(k), \cdots, e_{m}(k)\right]$

$$
\phi(k)=\left[\begin{array}{ccccc}
y_{1}^{T}(k) & \bar{u}_{1}^{T} & 0 & \cdots & 0 \\
y_{2}^{T}(k) & 0 & \bar{u}_{2}^{T} & \cdots & 0 \\
\vdots & \vdots & \vdots & \ddots & \vdots \\
y_{m}^{T}(k) & 0 & 0 & \cdots & \bar{u}_{m}^{T}
\end{array}\right]
$$

Among

$$
\begin{aligned}
& y_{i}=\left[-y_{i}(k-1), \hat{y}_{i}(k-2), \cdots, f y_{i}\left(k-n_{a}\right)\right]^{T} \bar{u}_{i}=\left[\bar{u}_{i 1}^{T}(k), \bar{u}_{i 2}^{T}(k), \cdots, \bar{u}_{i r}^{T}(k)\right]^{T} \\
& u_{i j}(k)=\left[u_{j}\left(k-d_{i j}, u_{j}\left(k-d_{i j}-1\right) \cdots, u_{j}\left(k-d_{i j}-n_{b i j}\right)\right]^{T} i=1,2, \cdots, m ; j=1,2, \cdots, r\right.
\end{aligned}
$$

Then (7) can be written are

$$
y(k)=\phi(k) \theta+e(k)
$$

In the formula(9) is the colored $e_{i}(k)$ noise, the unbiased consensus estimate of systen parameters can be acquired by maximum likelihood method or extended least squares method; however, $e_{i}(k)$ if is very small, it can be approximated as white noise process. This time, $e_{i}(k)$ instead of the above method with least squares to parameter estimation, the following method used is the least squares $[15]$

It is seen by the least squares method, least squares parameter estimation formula of multiple-input multiple-output system is:

$$
\left\{\begin{array}{c}
\hat{\theta}(k)=\hat{\theta}(k-1)+K(k)[y(k)-\phi(k) \hat{\theta}(k-1)] \\
K(k)=P(k-1) \phi^{T}(k)\left[I_{m}+\phi(k) P(k-1) \phi^{T}(k)\right]^{-1} \\
P(k)=[I-K(k) \phi(k)] P(k-1)
\end{array}\right.
$$

MIMO system recursive least squares estimation steps is showed in Figure 1 


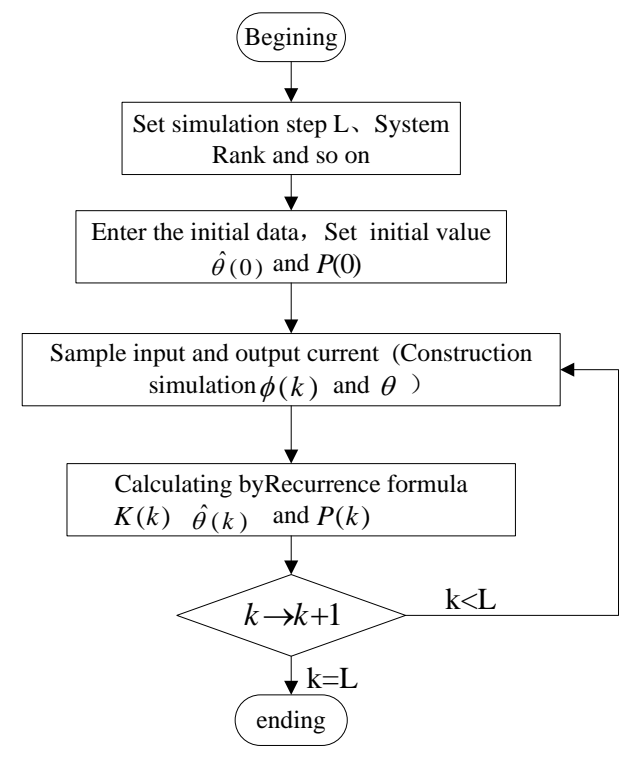

Figure 1. Flow Chart Of Recursive Least Squares Parameter Identification

\section{Parameter Identification Simulation of Synchinonous Generator}

In the above state equation of multiyariate synchronous generator [16-17], the parameters will be identified is

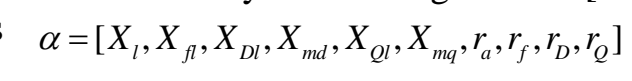

A100MW synchronous generator with thefollowing parameters (per unit):

$$
\begin{aligned}
& X_{d}=1.0 \quad X_{a d}=0.85 X_{f}=1.03 \quad X_{D}=0.95 \quad X_{q}=0.6 \quad X_{a q}=0.45 \\
& X_{Q}=0.7 \quad r_{a}=0.005 \quad r_{f}=0.000656 \quad r_{D}=0.00151 \quad r_{Q}=0.00159
\end{aligned}
$$

Parameters can be exported:

$$
X_{l}=0.15 \quad X_{f l}=18 \quad X_{D l}=0.10 \quad X_{Q l}=0.25 \quad X_{m d}=0.0427 \quad X_{m q}=0.0776
$$

Substituting the paraneters into the formula (5) that it can be given:

$$
A(\alpha)=\left[\begin{array}{ccccc}
0.02385 & 0.0079 & 0.01423 & 1 & 0 \\
0.0011 & 0.00278 & 0.00156 & 0 & 0 \\
0.0043 & 0.00358 & 0.00865 & 0 & 0 \\
-1 & 0 & 0 & 0.016 & 0.01035 \\
0 & 0 & 0 & 0.0033 & 0.0044
\end{array}\right] \quad C(\alpha)=\left[\begin{array}{ccccc}
4.77 & 1.58 & 2.85 & 0 & 0 \\
-1.58 & -4.238 & -2.37 & 0 & 0 \\
0 & 0 & 0 & 3.218 & 2.07
\end{array}\right]
$$

$$
G(s)=\frac{1}{A(s)}\left[\begin{array}{l}
B_{11}(s) \\
B_{21}(s) \\
B_{31}(s)
\end{array}\right]
$$

Where in $A(s)=1.0000 \mathrm{~s}^{5}-0.0557 s^{4}+1.0010 s^{3}-0.0158 s^{2}+0.0001 s-0.0000$

$$
\left\{\begin{array}{l}
B_{11}(s)=9.2000 s^{4}+4.5326 s^{3}+4.4191 s^{2}-0.0286 s+0.0000 \\
B_{21}(s)=-8.1880 s^{4}-1.2585 s^{3}-6.6177 s^{2}+0.0573 s-0.0001 \\
B_{31}(s)=5.2880 s^{4}-3.4117 s^{3}+2.0443 s^{2}-0.0235 s+0.0000
\end{array}\right.
$$

The input amount provided is not relevant white noise with unit variance, output is uncorrelated white noise of 0.1 variance, remove mean $P(0)=10^{6} I, \hat{\theta}(0)=0$, the recursive 
least squares algorithm can be used to derive the unbiased consensus estimate of the unknown parameters [18].

Figure 2, Figure 3, Figure 4, Figure 5 shows the respective parameter identification result of transfer function model. The parameters obtained by the identification results can get parameter of state equation through of mutual conversion, we substituted it into synchronous generator voltage equation (5)of the magnetic chain variable, we can be obtained result of parameter identification, as shown in Table 1.

By recognition result, we will be estimating for each to be identified parameter with recursive least squares method, the resulting error of identification value and the true value of is not more than $3.3 \%$, the result meet the requirements of errors. It shows the validity of the method.

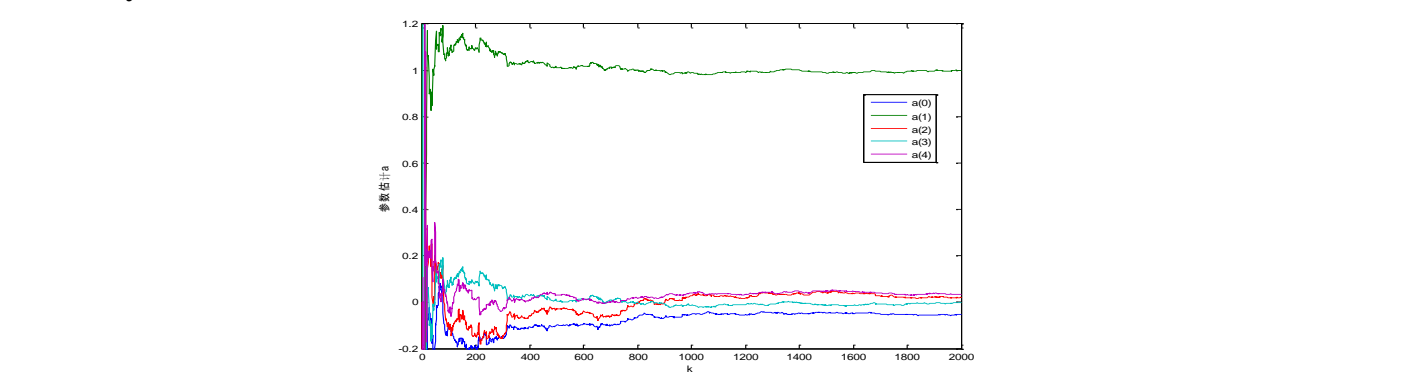

Figure 2. The Recognition Result of $A(s)$

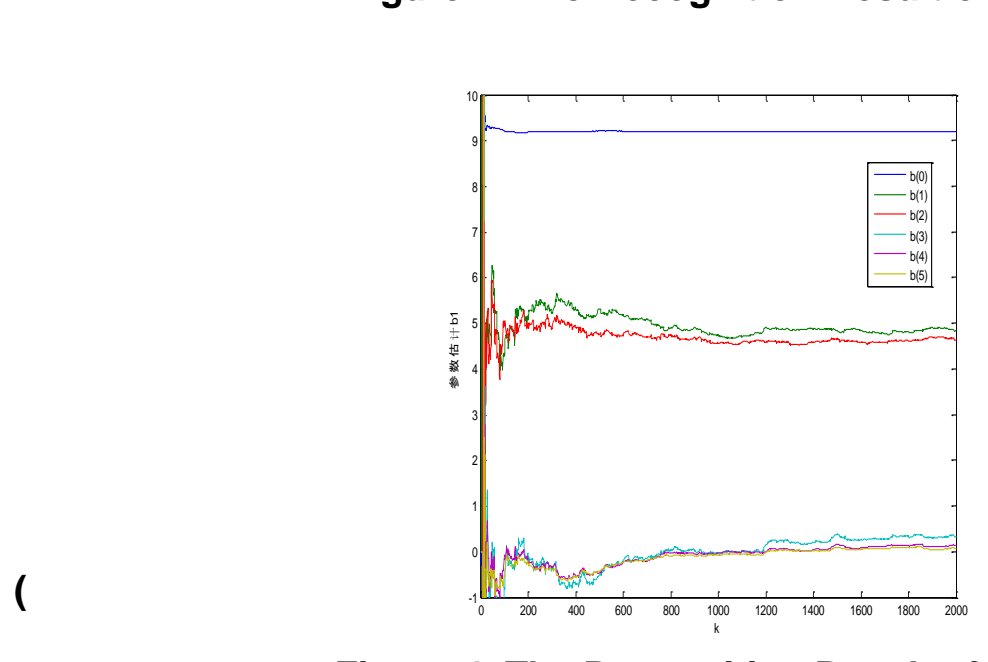

Figure 4. The Recognition Result of $B_{11}(s)$
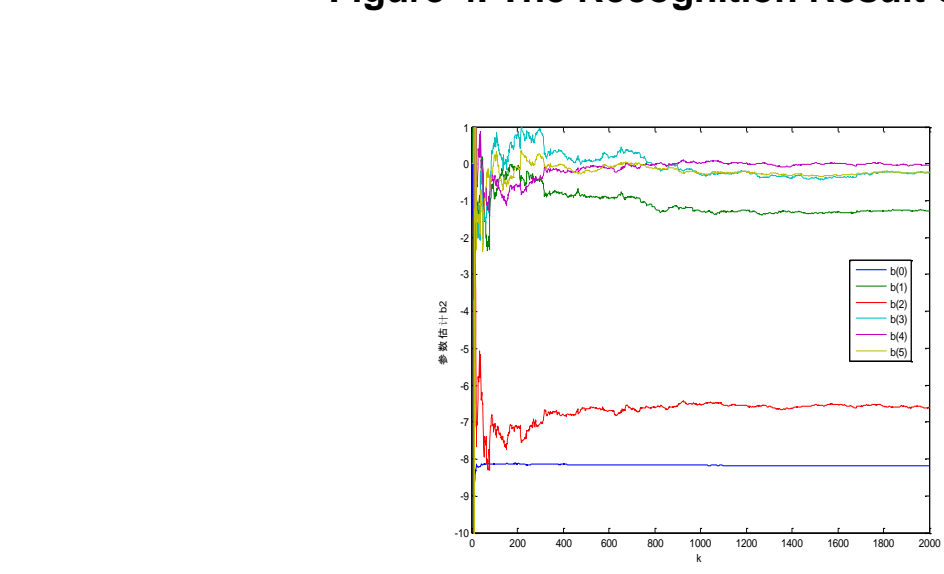

Figure 4. The Recognition Result of $B_{21}(s)$ 


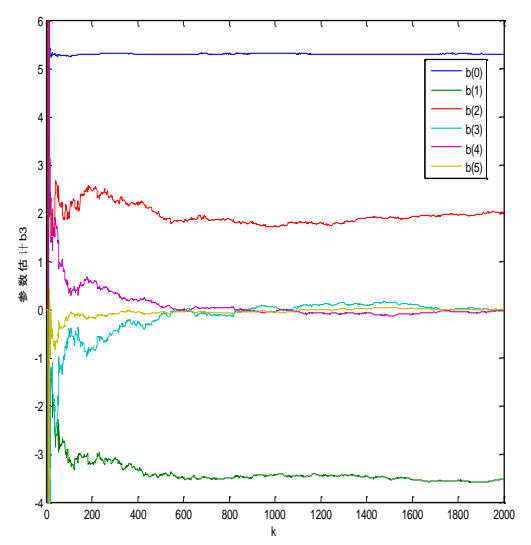

Figure5. The Recognition Result of $B_{3 I}(s)$

Table 1. The Identified Parameter

\begin{tabular}{|c|c|c|c|}
\hline parameter & true value & identified value & error $(\%)$ \\
\hline$X_{l}$ & 0.15 & 0.1512 & 0.8 \\
\hline$X_{f l}$ & 0.18 & 0.1745 & 3.1 \\
\hline$X_{D l}$ & 0.10 & 0.1021 & 2.1 \\
\hline$X_{m d}$ & 0.0427 & 0.0425 & 0.5 \\
\hline$X_{Q l}$ & 0.25 & $\mathbf{X} 2545$ & 1.8 \\
\hline$X_{m q}$ & 0.0776 & 0.0795 & 2.5 \\
\hline$r_{a}$ & 0.005 & 0.00514 & 2.8 \\
\hline$r_{f}$ & 0.000656 & 0.000671 & 2.3 \\
\hline$r_{D}$ & $0.9015 \%$ & 0.001554 & 2.9 \\
\hline$N_{O}$ & 0.00159 & 0.001643 & 3.3 \\
\hline Maximum error $(\%)$ & 0 & - & 3.3 \\
\hline
\end{tabular}

\section{Conclusion}

Aiming the problem that identified parameters are more of synchronous generator model, The state space model was derived on the basis of the synchronous generators Park model, and the derivation steps of the recursive least squares algorithm wasgiven. Theory and simulation results showed that the principle of parameter identification of the state equation of synchronous generator model using the recursive least squares algorithm is simple and easy to understand, clear of physical processes, good of identify effect. However, due to variable of state equation based on park model is more, the approximation linear perocess creating a greater error between the measurement equation and the state equation, coupled with the algorithm itself need also to be improved, which directly affected the accuracy of identification. Recursive algorithm can be improved or added forgetting factor, this will further improve the recognition accuracy and authenticity. 


\section{References}

[1] L. Sun, "Parameters identification based on Park Model of synchronous generator", Proceedings of the CSEE, vol.29, no.19, (2009),pp.50-56.

[2] T. Jiang, "The convergence of multi-value improving method of Least Squares parameters Identification of synchronous motor", Electric Machines and Control, vol.4, no. 2, (1999), pp.203-206.

[3] M. Qiang and J. Zhang, "Recursive least squares method parameter identification and Simulation based on MATLAB", Automation \&Instrumentation, No.6, (2008), pp.4-5.

[4] C. Du, S. Liu and K. Zhao, "A Dsp-Based Fast Tracking Resolver-to-Digital Conversion method",ControlEngineeringof China, vol.17, no. 1, (2010), pp.35-41.

[5] C. Wang, X. Qian, K.Yu and Z. Jiang, "Based on recursive least squares method of permanent magnet synchronous motor parameters identified", Torpedo technology ,no.6, (2013), pp.31-35.

[6] W. Li and X. Zhang, "Study of Solid Rotor line-start PMSM Operating Performance", IEEE Trans. on Magn, vol.45, no. 10, (2009).

[7] T. Ding, N. Takorabet and F. M. Sargos, "Design and analysis of different line-start PM synchronous motors foroil-pump application", IEEE Trans.OnMagn, vol.45, no.3, (2009).

[8] K. Kurihara and M.A. Rahman, "High-performance line-start permanent-magnet synchropous motors synchronous motors", IEEE Trans. On Magn, vol.40, no.30, (2004).

[9] A. Mahmoudi and I. Mejri, "Analysis of conduction-radiation heat transfer with vanable thermal conductivity and variable refractive index:application of the 1attice boltzmann method", International Journal of Heat and Technology,vol.33, no.1, (2015), pp.1-8.

[10] F. Corvaro*, G. Nardini*, M. Paroncini* and R. Vjtali* "PrV and numerical analysis of natural convective heat transfer and fluid flow in a square cavity with two vertical obstacles", International Journal of Heat and Technology, vol.33, no.2, (2015), pp.51-56.

[11] Z. D. Liu, "Study of identification and Simulation of hip Synchronous Generator", Harbin Engineering university, Harbin, (2011).

[12] H. Zhou and B. Xie, "Permanent magnet symchronous motor design of individual pitch", Small Motor Technology Conference Proceedings of the Eighteenth China, Beijing, (2013).

[13] Z. Pang, "Simulation of System identification and adaptrve control based on MATLAB", BeiHang university Press, Beijing, (2009).

[14] K. Wang, "Power System Analysis ', China Electric Power Press, Beijing, (2008).

[15] J. Ding, "Dynamic Parameter Identification of Synchronous Generator", wuhan university, wuhan, (2002).

[16] S. Wang, "Parameter Identification of Permanent Magnet Synchronous Motor and Control Strategy", Beijing Jiaotong university, Beijing, (2011),

[17] H. Zhang, "Strategies for Permanent Magnet Synchronous Motor of Frequency conversionDrives and Robust Control" Chira univetsity of Mining \& Technology, Beijing, (2011).

[18] C. Liu, C. Zhang. S. Feng and L. Liu, "Interior permanent magnet synchronous motor parameter identification technology", Electric Machines \& Control Application, Beijing, (2014).
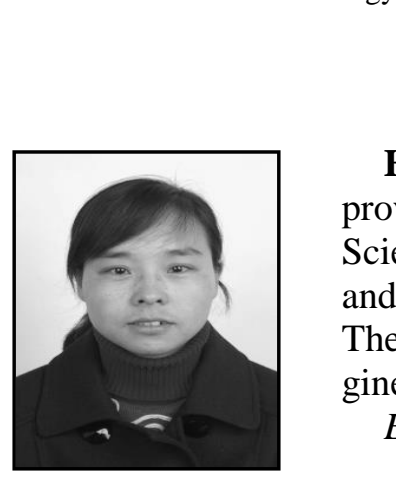

\section{Authors}

Hui-Ying Huang, she was born in1978 in Luoyang city henan province, in 2009 graduated from Zhengzhou University, Master of Science; She is a lecturer in Department of Electrical Engineering and Automationof Luoyang Institute of Science and Technology. The main research interest is image processing, electronicen gineering.

\section{E-mail:hjfhhyl@163.com}

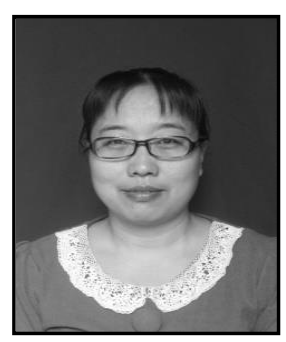

Juan-Mei Zhang, she was born in1974 in Sanmenxia city henan province, in 2005 graduated from Henan University of Science and Technology, Master of Science; She is a lecturer in Department of Electrical Engineering and Automation of LuoyangInstitute of Science and Technology. The main research interest is Power Electronics, Control theory \&Control Engineering.

E-mail:smxjixin@163.com 


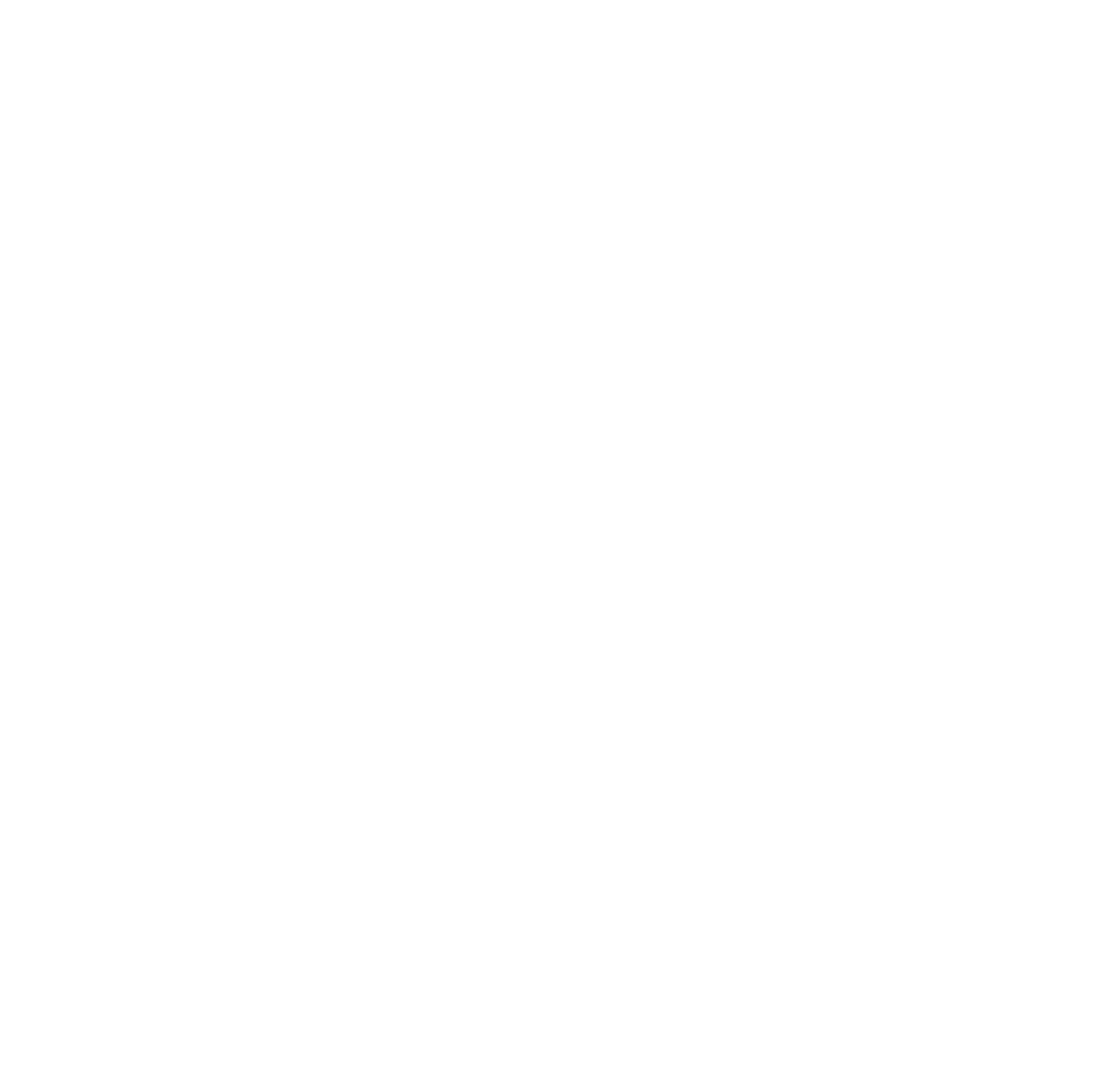

